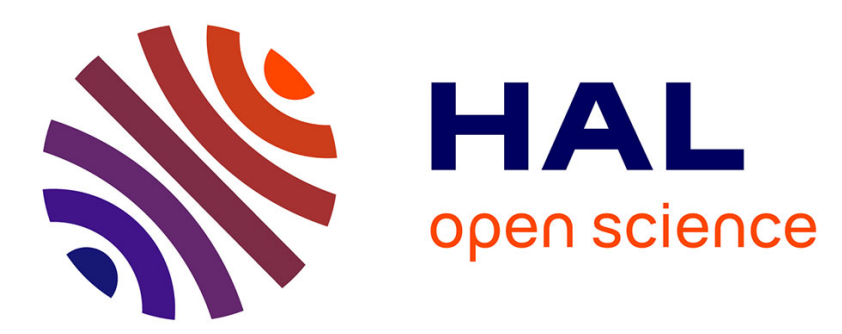

\title{
Using of tensegrity grid dynamic behavior to identify its self-stress level
}

\author{
Jean-François Dubé, Nicolas Angellier
}

\section{To cite this version:}

Jean-François Dubé, Nicolas Angellier. Using of tensegrity grid dynamic behavior to identify its self-stress level. Journal of Constructional Steel Research, 2013, 90, pp.133-139. 10.1016/j.jcsr.2013.07.026 . hal-01012043

\section{HAL Id: hal-01012043 \\ https://hal.science/hal-01012043}

Submitted on 31 Mar 2016

HAL is a multi-disciplinary open access archive for the deposit and dissemination of scientific research documents, whether they are published or not. The documents may come from teaching and research institutions in France or abroad, or from public or private research centers.
L'archive ouverte pluridisciplinaire HAL, est destinée au dépôt et à la diffusion de documents scientifiques de niveau recherche, publiés ou non, émanant des établissements d'enseignement et de recherche français ou étrangers, des laboratoires publics ou privés. 


\title{
Using of tensegrity grid dynamic behavior to identify its self-stress level
}

\author{
Jean François Dubé ${ }^{\mathrm{a}, *}$, Nicolas Angellier ${ }^{\mathrm{b}}$ \\ a LMGC-UMR 5508, Place E. Bataillon, F-34095 Montpellier cedex 5, France \\ b Université de Limoges, GEMH GCE'D, 19300 Egletons, Limoges, France
}

\begin{abstract}
The structures of tensegrity are light structures of which stability is conditioned in a state of prestressing called self-stress. Work presented relates to a structure with double layer which is instrumented with gauges of defor-mation to determine the forces in the bars. The aim of work is to find the forces in the elements by indirect mea-surement for further in-situ applications when the bars instrumentations will be impossible. Here, we explore the possibility of using the measurement of the fields of acceleration of the nodes of a structure of tensegrity under dynamic loading in order to identify the self-stress state. We show the effect of the self-stress level on the modes of vibration of the structure to determine the correlation between the precision of this identification and the influence of the mode concerned. The results confirm the complex relation which exists between the Eigen-modes and the identification of self-stress state. The identification is generally more powerful for the first global modes which are impacted by all self-stress states than for the local modes which are often dependent only of one self-stress state.
\end{abstract}

Keywords:

Tensegrity

Self-stress

Acceleration measurement

Inverse analysis

\section{Introduction}

Tensegrity structures are light space structures made up of compressed bars and tended cables which ensure continuity. The rigidity of the tensegrity structure is function of the elements stiffness but also of the cables tensions. This state called self-stress state ensures the rigidity of the structure and its stability. The knowledge of this self-stress state is essential for the structure safety in place.

The continuity of the tended elements implies that the degradation of one element can influence all or part of the structure. The degradation of one element can modify the stiffness of the structure. Therefore, it is necessary to identify the damage state of the elements. Here, we suppose that damage is related to the force in the element. It is not reasonable to measure the internal force of all elements during the life of the work. We must identify the internal forces using an indirect measurement and an inverse analysis [1,2].

Among the measurements which can be carried out easily, displacement fields and Eigen-frequencies of the structure are widely used [3-7]. We chose to use the nodes displacements of the structure under static or dynamic loading $[8,9]$. Tests and numerical simulations made it possible to show the relevance of the procedure to find the weight parameters of the elementary self-stress states: we showed that we

\footnotetext{
* Corresponding author. Tel.: +33 4671496 40; fax: + 33467144555 . E-mail addresses: jean-francois.dube@univ-montp2.fr (J.F. Dubé), nicolas.angellier@unilim.fr (N. Angellier).
}

were able to find the tension of cables which have a loss of tension of $25 \%$ compared to their design state [10].

The method is the same as the one for the optimization of structures $[11,12]$. For a tensegrity system, the number of unknown factors is a function of the number of cables. This number becomes important for complex structures. One can apply a load to the structure to determine his Eigen-frequencies for his lifetime, however, one cannot obtain as many Eigen-frequencies of cables. The identification based on the only Eigen-frequencies implies to limit the number of unknown parameters. To carry out the identification, we can use the evolution of the acceleration field of the structure [9] to obtain frequential and deformation information. However, this method always does not converge towards the desired solution because we have sometimes too much information. It must be more judicious to have a strategy using the most effective modes.

To characterize the structure by non-destructive dynamic tests, we work on his vibratory behavior. We study the influence of self-stress level imposed on the dynamic behavior of the structure. The structural design of tensegrity leads to a mechanical nonlinear behavior which one finds in the evolution of the Eigen-mode. This study includes numerical simulations on a small plane tensegrity grid with double layer to correlate modeling and tests. The numerical simulations corroborate the test results with a nonlinear evolution of the Eigenfrequencies of the first two modes increasing with the self-stress level.

To go further, the grid is subjected to sinewave excitation for a given frequency. It appears that some frequencies allow a better identification than others. This study tries to establish a relation between Eigen-mode, self-stress state, and the electiveness of the identification. Numerical 


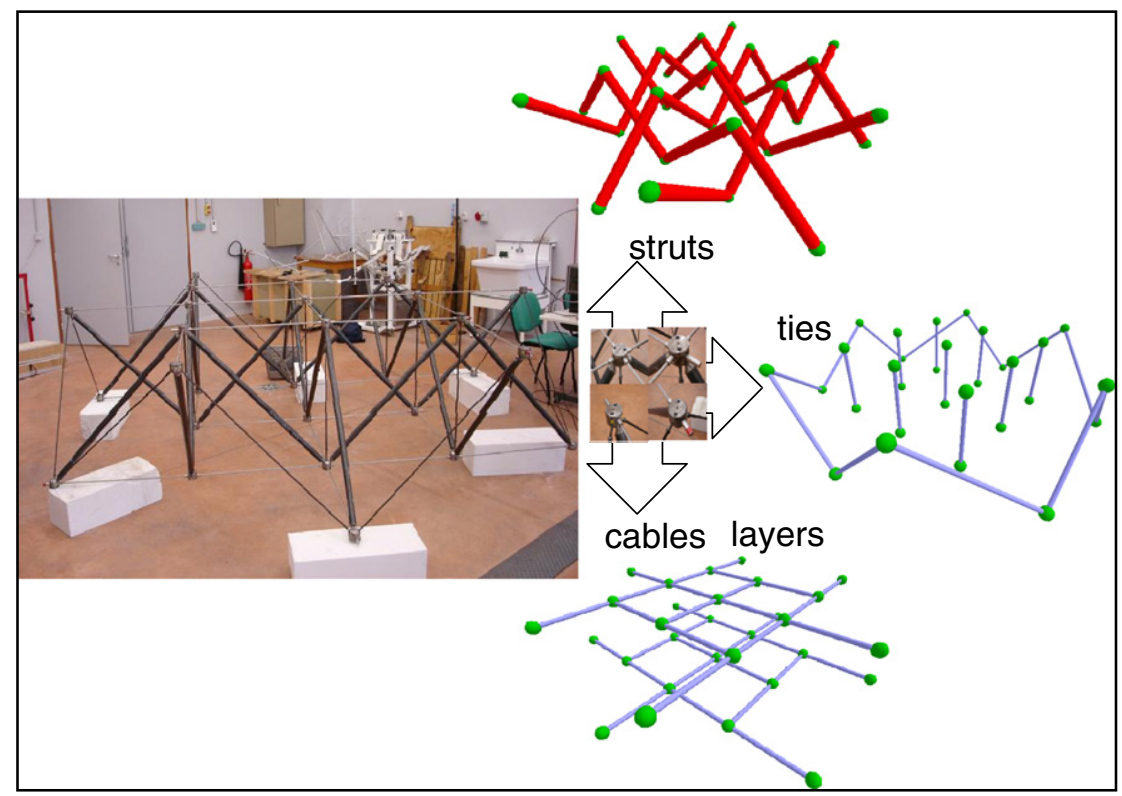

Fig. 1. Mini grid, modeling.

simulations validate the method proposed here for the identification of such a tensegrity grid. This article shows the importance of the choice of the mode of excitation used for identification for the quality of the results.

\section{Internal state of the minigrid}

\subsection{Mini grid}

This is a double layer plane grid of cables the basic pattern of which uses the expander principle: two by two, the struts form perpendicular, opposing V's, separated by a vertical tie the length of which can be made to vary. This consists of 81 components linked by nodes: 24 compressed struts (tubes) and tensioned elements, i.e., 36 cables in addition to 9 vertical ties and 12 peripheral ties; the ties are the active components of the structure (Fig. 1). This grid has been designed to match to the definition of a tensegrity structure: "system in a state of self-stable equilibrium (self-stress here), which includes a discontinuous set of compressed components (bars) inside of a continuum of tensioned components (cables and ties)" (Motro [13]).

\subsection{Self-stress state}

System equilibrium is obtained when all nodes are in equilibrium. The static equilibrium of a node $I$ is written as:

$\sum_{j \neq i} T_{i j}+F_{i}=0$

$T_{i j}$ is the internal force vector of the element linking node $j$ to node $i$, and $F_{i}$ is the external force vector directly applied to node $i$. The internal forces $T_{i j}$ are a function of the deformation of each element; $l_{i j}^{0}$ is the length of the element connecting nodes $i$ and $j$ in the reference configuration.

The projection of the equilibrium equation on three axes can be simplified by introducing the force density $q_{i j}$ of each element $(i, j)$ :

$q_{i j}=\frac{T_{i j}}{l_{i j}^{0}}$
The system of equations obtained by applying to all the nodes of the structure is:

$A q=\boldsymbol{f}$

with $\mathbf{A}$ for the equilibrium matrix of the structure (dimension $b \times 3 n$ ), $\mathbf{q}$ for the vector describing the force densities in elements $b$, and $\mathbf{f}$ for the vector of the external forces acting on nodes $n$.

Self-stress is the whole set of internal forces occurring in the initial state, without external loading. This state of equilibrium corresponds to a field of force densities $\mathbf{q}_{0}$ satisfying:

$A \boldsymbol{q}_{\boldsymbol{o}}=\boldsymbol{0}^{\Leftrightarrow} \boldsymbol{q}_{\boldsymbol{0}} \quad \operatorname{ker} \boldsymbol{A}$

The self-stress state can be expressed on the basis of the subspace kerA. This base, denoted $S$, is composed of several basic self-stress states. There are 2 kinds of basic state: total if it solicits all the system's components, partial otherwise. The base $S$ must allow building a total selfstress state. The self-stress basis $\mathbf{S}$ resulting from kernel $\mathbf{A}$ cannot be used directly, since the equilibrium matrix A does not take into account the unilateral behavior of elements. Every self-stress state that meets the unilaterality conditions for tensioned members is called "conform." A conform self-stress state can result from a linear combination of several conform states (Sanchez [14]), or directly from linear combination of the elements defining $\mathbf{S}$ (Quirant et al. [15]):

$\boldsymbol{q}_{0}=\boldsymbol{S} \boldsymbol{\alpha}$

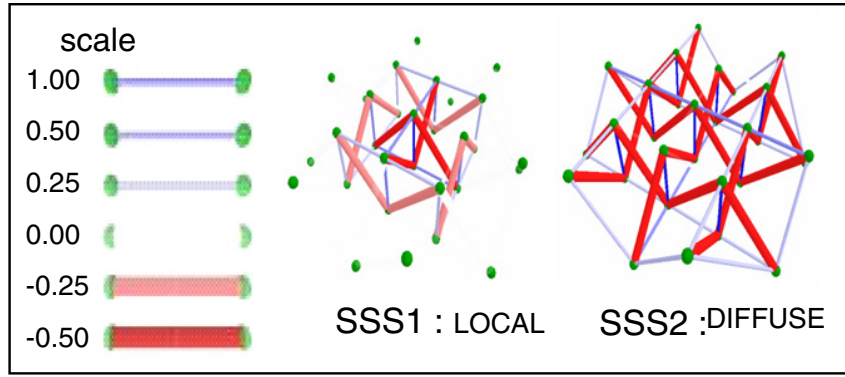

Fig. 2. Mini grid basic self-stress states. 


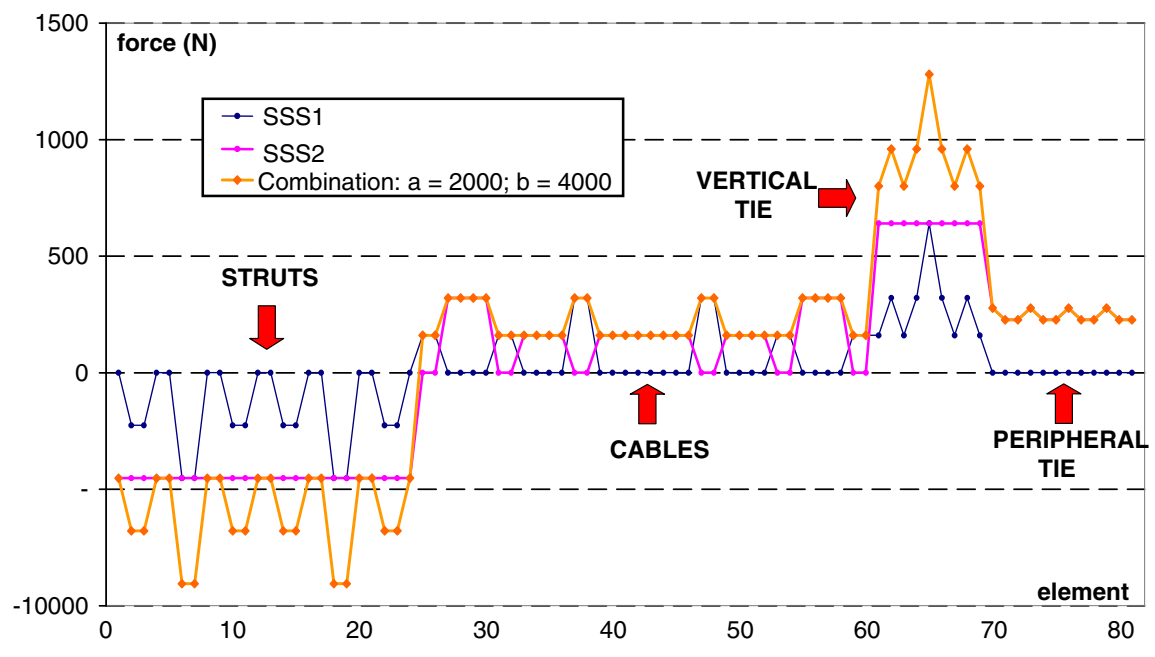

Fig. 3. Combination of force vectors of the mini grid for a realistic state.

The components of the $\boldsymbol{\alpha}$ vector are chosen to satisfy stress conditions for members.

\subsection{Mini grid self-stress state}

Tenségrité2000 software (Quirant [16]) determines the basic selfstress states of a tensegrity structure starting from:

- boundaries conditions: three non-symmetrically support conditions here,

- geometry: nodes position and components connectivity.

Thus, the mini grid has only two basic self-stress states: a local self stress state SSS1 and a diffuse self stress state SSS2. Fig. 2 shows that for the state SSS1, peripheral elements are not involved in the equilibrium (in white), while for the state SSS2, this concerns only few cables. By multiplying these basic states by the elements free lengths $l_{i}$, we obtain the vectors of internal force [Ti] $=\operatorname{SSSi} l_{i}$. The two vectors [T1] and [T2] calculated can be combined with 2 independent weighting coefficient $a$ and $b$. The linear combination $a[\mathrm{~T} 1]+b[\mathrm{~T} 2]$, enables to build a total self-stress state which ensures the stability of the structure and the service limit states that we impose.

Fixing the ratio $a / b$, the parameter $a$ is used as the indicator of selfstress level of this selected target state for the studied structure. This global level is made to vary to achieve realistic values of forces in the elements which permit to have a structure rigid enough without reaching the struts buckling. A ratio of 0.5 provides the most homogeneous distribution of forces possible in the components, particularly for cable tensions; elements that pilot the rigidity of the structure: for example where $a=2000$ (Fig. 3).

\subsection{Standard identification of internal state}

To identify the internal state $\alpha_{i d}$ of the grid we usually employ a method consisting in the measure of forces in a limited number of elements $\left\{T_{\text {mes }}\right\}$, using dedicated strain gauges. We wedge on the outcome of these measures a state generated by the self-stress base (formed by the force vectors [Ti] previously determined). For this, we employ the pseudo-inverse method to minimize the difference between the generated state and the measured state (Averseng [17]). This initial identification of the self-stress of the grid, established during its tensioning and which serves as a reference, gives coefficient $a$ and $b$, i.e. a ratio $a / b$ close to the target ratio. The maximum inaccuracy on the forces leads to a maximum error on the identified coefficient $a$ which corresponds to an accuracy lower than $5 \%$ on the state of selfstress.

\section{Relation between Eigen-modes and self-stress}

This section shows the influence of self-stress state on the variation in Eigen-modes of the tensegrity structure.

\subsection{Eigen-modes}

The stiffness of the grid is conditioned by the self-stress. It is, therefore, obvious that self-stress influences the Eigen-modes of the structure. The structure is set in self-stress, with the same boundaries conditions, for the combination of the two elementary states SSS1 and SSS2, using the CAST3M finite elements code ([18]).

To calculate a lattice, the usually raised hypothesis is a bar-type behavior for modeling all elements of a tensegrity structure. In our case, this assumption is challenged by a previous study (Dubé et al. [19]) where we detected the bending in the struts in a self-stress state. We showed that, in static and dynamic, compressed elements and ties had a beam-type behavior. The cables are modeled by bar elements.

We have chosen two combinations which meet mechanical requirements and as the base for a sensitivity study:

$\boldsymbol{q}_{\text {ref_h }}=\boldsymbol{S} \boldsymbol{\alpha}_{\text {ref_h }}$ with $\boldsymbol{\alpha}_{\text {ref_h }}=\{2000 ; 2000\}$, «homogeneous» selfstress state. This corresponds to a ratio $a / b=1$. Thus we can compare the influence of each weighting coefficient on Eigenfrequencies, conserving realistic values for forces in the elements.

$\boldsymbol{q}_{\text {ref_r }_{\mathbf{r}}}=\boldsymbol{S} \boldsymbol{\alpha}_{\text {ref_r } \mathbf{r}}$ with $\boldsymbol{\alpha}_{\text {ref_r }_{\mathbf{r}}}=\{2000 ; 4000\}$, "realistic» self-stress state with a ratio $a / b=0.5$. The maximum force in elements is lower than: their elastic yield, the screw thread strength of the anchorage nodes and the force-anchorage slip of the layer cables.

With thereby defined self-stress states we are able to compute the first five Eigen-modes of the structure: the Eigen-frequencies are given in Table 1. It is noted that the first two modes have frequencies close to $5 \mathrm{~Hz}$ while the three following has frequencies about $30 \mathrm{~Hz}$. Between the homogeneous state and the realistic state, the frequencies

Table 1

Characteristics of the modes according to the self-stress state.

\begin{tabular}{|c|c|c|c|c|c|c|}
\hline State & Mode & 1 & 2 & 3 & 4 & 5 \\
\hline $2000 ; 2000$ & frequencies $(\mathrm{Hz})$ & 4.056 & 5.619 & 29.824 & 31.042 & 31.187 \\
\hline $2200 ; 2000$ & & 4.076 & 5.622 & 29.826 & 31.042 & 31.186 \\
\hline $2000 ; 2200$ & & 4.103 & 5.637 & 30.399 & 31.963 & 32.134 \\
\hline $2000 ; 4000$ & & 4.496 & 5.783 & 32.002 & 39.050 & 37.840 \\
\hline $2200 ; 4000$ & & 4.514 & 5.786 & 32.006 & 39.053 & 37.843 \\
\hline $2000 ; 4200$ & & 4.537 & 5.798 & 32.063 & 39.676 & 38.077 \\
\hline
\end{tabular}




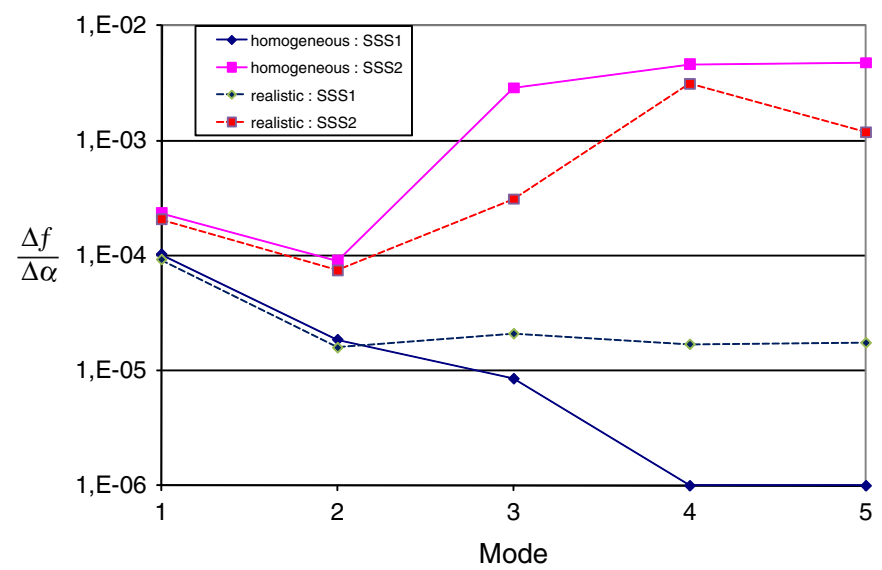

Fig. 4. Impact of the self-stress states on the Eigen-frequencies.

of modes 1 and 2 evolve very little while for modes 3, 4, 5 this evolution is sufficiently consequent to make their frequencies quite distinct. It is also noticed that there is an inversion of the frequencies for modes 4 and 5 .

To finely study the sensitivity of the Eigen-frequencies compared to two self-stress states SSS1 and SSS2, each weighting coefficient $\alpha$ ( $\alpha$ is here alternatively $a$ or $b$ ) applied to the self-stress basis is affected by an identical variation $\Delta \alpha=200$. This gives a relative variation of:

- $10 \%$ for the coefficients $a$ and $b$ initially to 2000 ;

$10 \%$ for the coefficient $a$ initially to 2000 and only $5 \%$ for the coefficient $b$ equal to 4000 .

The variation of each coefficient is independent from that of the other. Table 1 recapitulates values associated with the various Eigenmodes for the variation of each coefficient.

From these data we can compute the matrix of the frequency gradient compared with the variation of the weighting coefficient of the self-stress basis: $\frac{\Delta f}{\Delta \alpha}$

If the ratio is small, then a small variation of $\alpha$ implies a small variation of $f$. Thus, if the ration is large, then a small variation of $\alpha$ implies a large variation of $f$.

By taking the inverse of the ratio, we obtain the following relation:

$\frac{\Delta f}{\Delta \alpha}$ is small $\Rightarrow \frac{\Delta \alpha}{\Delta f}$ is large $\Rightarrow f$ gives a large influence on $\alpha$.

$\frac{\Delta f}{\Delta \alpha}$ is large $\Rightarrow \frac{\Delta \alpha}{\Delta f}$ is small $\Rightarrow f$ gives a small influence on $\alpha$.
Fig. 4 shows the ratio $\frac{\Delta f}{\Delta \alpha}$ for the two elementary self-stress states for the first five Eigen-frequencies.

One observes a difference in behavior very marked between the two states: while for state 1 the ratio is weak and decreases with the increase of the frequency, the tendency is reversed for state 2 with a ratio whose value is much higher.

We can note that for modes 1 and 2 , the variation $\frac{\Delta f}{\Delta \alpha}$ is not influenced by self-stress state compared to the other modes. We can suppose that the modes 1 and 2 have a large influence on the weighting coefficients of the self-stress states. We check if this relation which exists between the state of self-stress and the Eigen-modes influences the quality of the identification of the self-stress.

\subsection{Experimental validation}

To validate this numerical study, we have the results of an experimental study conducted on the minigrid structure (Dubé et al. [20]).

\subsubsection{Test conditions}

The minigrid is vertically excited by a vibrating pot positioned in boundary condition free/free on the higher layer of the structure (Fig. 5). Dynamic analysis is realized by harmonic analysis on a frequency range [ $0 \mathrm{~Hz} ; 100 \mathrm{~Hz}$ ], which is sufficient to identify the first mode of the structure. The sampling rate of the signals adopted is of $200 \mathrm{~Hz}$.

Three series of experiments are carried out to study the incidence of the self-stress on the dynamic behavior of the structure. The modification of the self-stress is obtained by the action on:

Serie 1: an active element of angle (with $0.49<a / b<2.8$ );

Serie 2: a peripheral, side active element (with $0.7<a / b<2.7$ );

Serie 3: 4 vertical active elements (with $a / b$ of about 0.11 ).

\subsubsection{Incidence of the self-stress on the first mode}

The analysis of the results is carried out while taking as indicator of the self-stress level, the coefficient $a$ local self-stress state. In series 1 and $2, a / b$ ratio varies from 0.5 to 3 , and we can note (Fig. 6) that the first Eigen-frequency increases with the level of the diffuse partial state (b) (while the parameter $a$ decreases). In series $3, a / b$ ratio remains appreciably constant, and the first Eigen increases with the level of the total self-stress state.

Simultaneously with the experiments, numerical simulations of the vibratory behavior of the structure were carried out. These simulations make it possible to compare on the one hand, the experimental results with results of simulation, and on the other hand, to sweep levels of self-stress not reached by the experiment (Fig. 6). Numerical
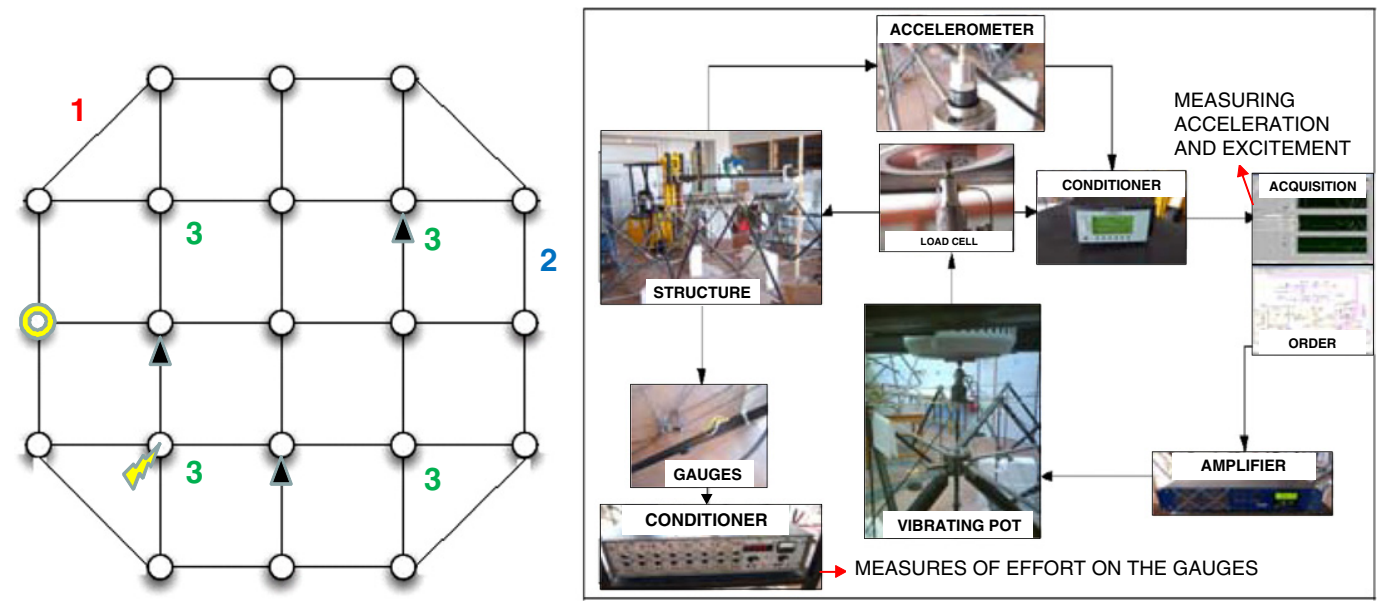

Fig. 5. Elements requested for each series of tests $(1,2.3)$ - nodes concerned with the excitation (flash), measurement (round) and the supports (triangles). 


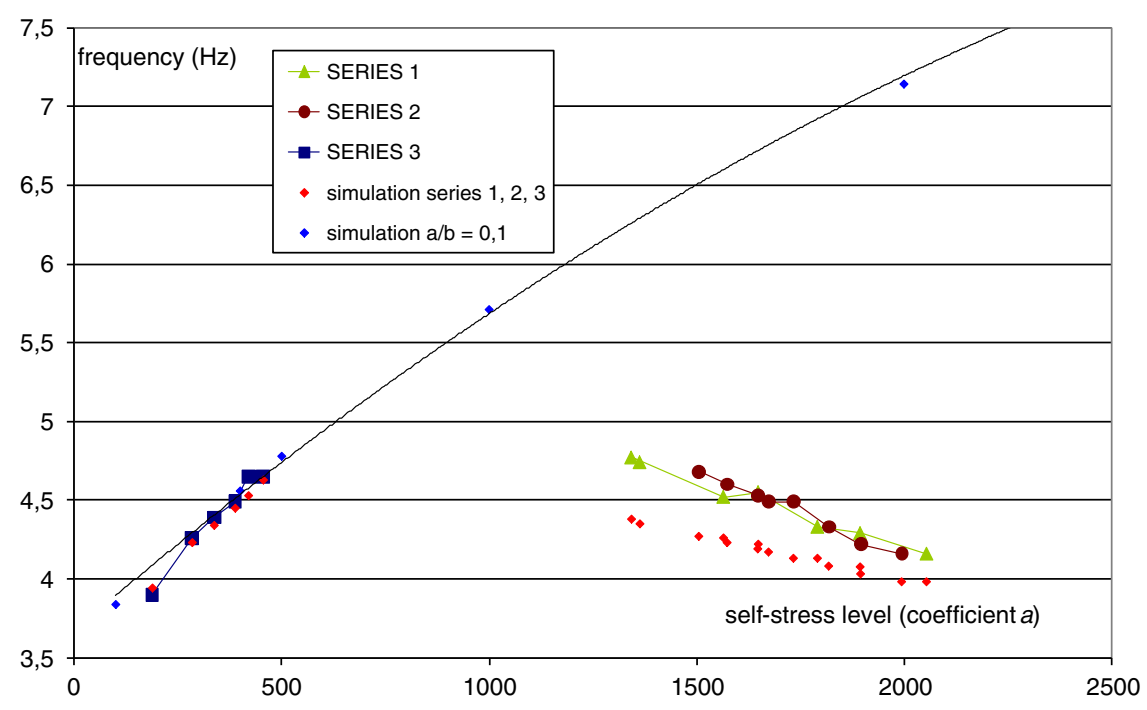

Fig. 6. Comparison of the first Eigen frequencies measured and simulated.

simulations use the self-stress coefficients $a$ and $b$ identified by the experimental tests. The comparison between experimental frequencies and those given by simulations makes it possible to note a very good agreement between them. The maximum differences do not exceed $10 \%$, and are even $<3 \%$ in the case of series 3 (Fig. 6 ).

\section{Correlation between the self-stress state identification of the minigrid and Eigen-modes}

The study of the correlation between the Eigen-mode and the identification of the self-stress state of a double layer grid with middle size and six self-stress states gave mitigated results (Dubé et al. [21]). Indeed, the influence of the self-stress state on each Eigen-mode does not give the reciprocal one when one uses the Eigen-modes to identify the self-stress states. Moreover we must use a number of Eigen-mode more important than the number of state self-stress to carry out a correct identification. We start again here the same study with a structure having only two self-stress states.

\subsection{Self-stress identification}

We study the relationship between Eigen solicitation and the identification of self-stress. For identification we use the evolution of the acceleration field of the structure [9]. An initial self-stress state defined by the $\boldsymbol{\alpha}_{\text {ref }}$ vector constitutes the reference state. The minigrid is activated by a sinusoidal nodal force the frequency of which corresponds to one of the first five Eigen-modes. The initial self-stress state is defined on the basis of a linear elastic behavior and large displacements. We assume that the vibratory behavior produces, on the other hand, small displacements only. Acceleration of the nodes of the structure is calculated by means of the CASTE3M code. The acceleration field is used as comparative data for identification by inverse analysis.

Identification starts from a random self-stress state close to the reference state. The procedure of identification uses a LevenbergMarquardt algorithm [22-25], which combines Newton method and gradient method. The purpose of error function $\mathfrak{I}(P)$. Function $\mathfrak{I}$

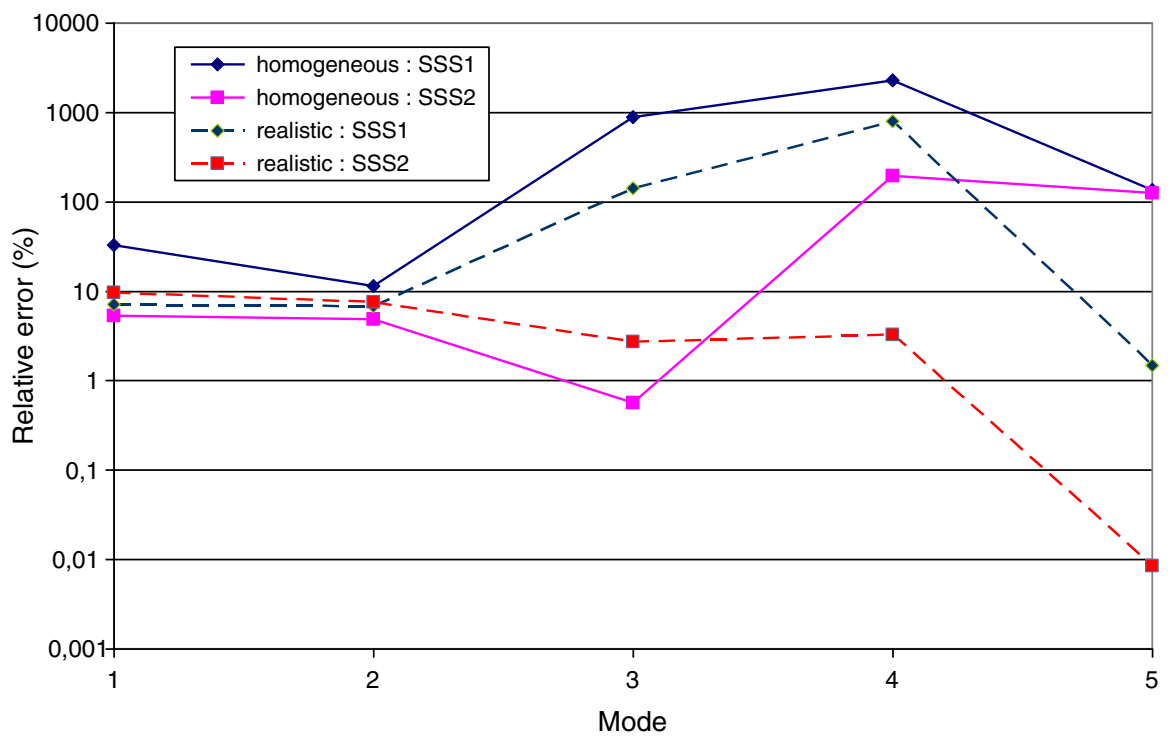

Fig. 7. Evolution of the quadratic error on the states parameters according to the calculated mode. 


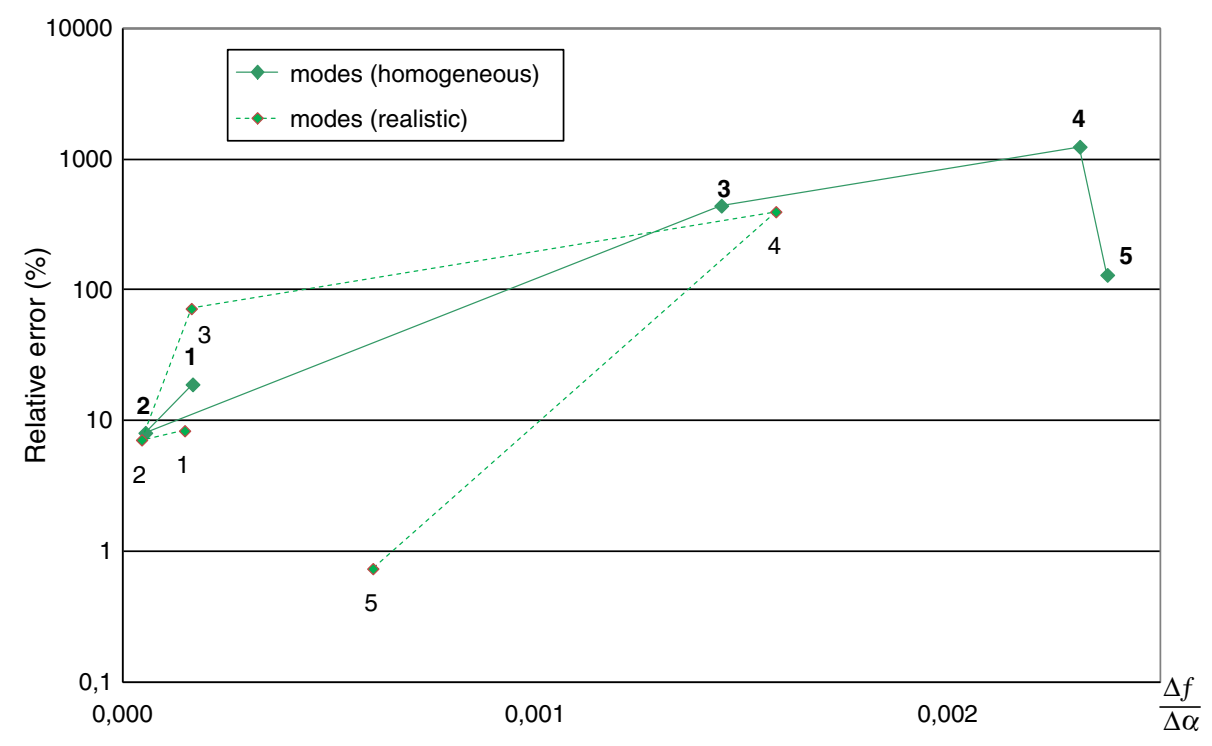

Fig. 8. Correlation between the relation frequency-self-stress state level and the identification error.

expresses the difference between simulated acceleration field and the acceleration field of reference:

$\left.\Im(P)=\frac{1}{2}\left[\frac{\left(R(P, t)-R_{\mathrm{ref}}(t)\right)}{\frac{\max \left(R_{\mathrm{ref}}\right)+\min \left(R_{\mathrm{ref}}\right)}{2}}\right)^{T}\left(\frac{\left(R(P, t)-R_{\mathrm{ref}}(t)\right)}{\frac{\max \left(R_{\mathrm{ref}}\right)+\min \left(R_{\mathrm{ref}}\right)}{2}}\right)\right]$

where $P$ is the vector of the parameters to be identified, $R$ the temporal response of the acceleration field of the simulated structure, $R_{\text {ref }}$ the response of the acceleration field of the structure to be identified.

The identified parameters are the weighting coefficients $a$ and $b$ of the elementary self-stress states. Identification is then carried out by taking account the difference between the response of the numerical structure and that of reference.

We consider the two following reference states:

«homogeneous»:\{2000; 2000\} (random self-stress state:\{3241.6; 2230\});

«realistic»: $\{2000 ; 4000\}$ (random self-stress state: $\{3241.6 ; 4460\})$.

Fig. 7 presents the evolution of the quadratic average of the relative error (difference between the reference coefficient and the identified one) associated with the two weighting coefficients for each identification.

The weighting coefficient of self-stress state 1 is less well identified than that of self-stress state 2 whatever the mode of vibration used for the identification. It is only for the first two modes that one arrives systematically to a good identification of the two self-stress states simultaneously with a suitable relative average error: about $10 \%$ what corresponds to a maximum force error lower than $1 \mathrm{kN}$ in the elements. On the other hand, in the realistic case, one observes that mode 5 conduct to an error relative even weaker for the two self-stress states and lower than $1 \%$ on average (the maximum force error is lower than $200 \mathrm{~N}$ in the elements). This result is rather curious compared to the result of mode 4 . Indeed these two modes are local modes of vibration affecting elements different but not those from the local self-stress state. However, for mode 5, there is less elements in vibration than for mode 4 . We can suppose that the identification is carried out more effectively on these some elements; however that does not explain the reason for which the local self-stress state is also correctly identified.

On the evolution of the error relative according to the $\frac{\Delta f}{\Delta \alpha}$ ratio (Fig. 8), we can notice that in a general way, to obtain a small error, one needs a small ratio. This corresponds to the modes whose frequency influences much the state of self-stress. It is here clearly the case for modes 1 and 2.

The influence of the excitation frequency on the identification of the weighting coefficients of the self-stress state is certain. Only the use of modes 1 and 2 gives results correct and stable for the identification of the two coefficients $a$ and $b$ whatever the reference self-stress state. Finally, the correlation between the influence of the self-stress state on the Eigen-mode and the quality of the identification is not obvious.

\section{Conclusion}

The study that we carry out is principally numerical. We show the link between the self-stress and the first Eigen-modes.

The study uses a double layer tensegrity minigrid having two elementary self-stress states. Several self-stress configurations are studied: an ideal configuration for which the self-stress is definite homogeneous and a configuration resulting from the measures on the experimental prototype.

We show that the Eigen-frequency is more sensitive to the selfstress for a diffuse state than for a local state. The first modes of the structure are global modes, it is thus normal that the diffuse self-stress state affects more its modes than the local state. We check that the influence of the self-stress state on the Eigen-modes can be used in an inverse process to identify the self-stress states starting from the Eigenmodes. We see overall that if the self-stress has low influence on the Eigen-frequency, then the use of this Eigen-frequency leads to a better identification of the self-stress. We must verify these results for other structure typologies, for example for tensegrity rings which can have only one self-stress state and can be foldable.

\section{References}

[1] Averseng J, Crosnier B. Prestressing tensegrity systems - application to multiple self-stress state structures. Int J Struct Stab Dyn 2004;4(4):543-57.

[2] Averseng J, Crosnier B. Static and dynamic robust control of tensegrity systems. J Int Assoc Shell Spatial Struct 2004;45(3):169-74.

[3] Périé JN, Leclerc H, Roux S, Hild F. Digital image correlation and biaxial test on composite material for anisotropic damage law identification. Int J Solids Struct 2007;44(9):2863-87.

[4] Lu ZR, Liu JK. Identification of both structural damages in bridge deck and vehicular parameters using measured dynamic responses. Comput Struct 2011;89: 1359-405.

[5] Murakami H, Nishimura Y. Static and dynamic characterization of some tensegrity modules. J Appl Mech 2001;68:19-27.

[6] Ndambi JM, Peeters B, Maek J, DeVisscher J, Wahab MA. Comparison of techniques for modal analysis of concrete structures. Eng Struct 2000;22(9):1159-66. 
[7] Pritchard JI, Adelman HM, Haftka RT. Sensitivity analysis and optimization of nodal point placement for vibration reduction. J Sound Vib 1987;119:277-89.

[8] Van Den Abeele K, DeVisscher J. Damage assessment in reinforced concrete using spec tral and temporal nonlinear vibration techniques. Cem Concr Res 2000:30:1453-64.

[9] Maeck J, et al. Damage identification in reinforced concrete structures by dynamic stiffness determination. Eng Struct 2000;22:1339-49.

[10] Dubé JF, Crosnier B. Identification of cables slackening by analyzing the temporal response of the structure. In: Motro R, editor. IASS 2004, shell and spatial structure from models to realization; 2004 [8 pages in cdrom].

[11] Olhof N, Eschenauer H, Schnell W. Applied structural mechanics. structural optimization. Springer; 1997

[12] Gurdal Z, Haftka RT, Kamat MP. Elements of structural optimization. Kluwer Academic Publishers; 1993.

[13] Motro R. Tensegrity. Kogan Page Science; 2003

[14] Sanchez LR. Contribution à l'étude mécanique des systèmes de tenségrité, 2. Université Montpellier; 2005 [Thèse de doctorat].

[15] Quirant J, Kazi Aoual MN, Laporte R. Tensegrity systems: the application of linear programmation in search of compatible self-stress states. J Int Assoc Shell Spatial Struct 2003;44(1):33-50.

[16] Quirant J. Systèmes de tenségrité et autocontrainte: qualification, sensibilité et incidence sur le comportement, 2. Université Montpellier; 2000 [Thèse de doctorat].
[17] Averseng J. Mise en œuvre et contrôle des systèmes de tenségrité, 2. Université Montpellier; 2004 [Thèse de doctorat].

[18] Verpeaux P, Charras T, Millard A. Castem 2000, une approche moderne du calcul des structures. In: Fouet JM, Ladevèze P, Ohayon R, editors. Calcul des structures et intelligence artificielle; 1988.

[19] Dubé J-F, Angellier N, Crosnier B. Comparison between experimental tests and numerical simulations carried out on a tensegrity mini grid. Eng Struct 2008;30(7):1905-12.

[20] Dubé JF, Crosnier B, Angellier N. Caractéristiques dynamiques d'une structure de tenségrité en fonction de son niveau d'autocontrainte. 19ème Congrès Français de Mécanique, Marseille; 2009 [6 pages in cdrom].

[21] Dubé J-F, Angellier N. Correlation between eigenmodes and the self-stress state identification of a tensegrity grid. Int J Space Struct 2006;21(4):223-32.

[22] Levenberg K. A method for the solution of certain nonlinear problems in least squares. Q Appl Math 1944;2:321-8.

[23] Luenberger DG. Introduction to linear and nonlinear programming. Addison-Wesley; 1984

[24] Marquardt DW. An algorithm for least squares estimation of nonlinear parameters. SIAM J Appl Math 1963(11):431-41.

[25] Walter E, Prontazo L. Identification de modèles paramétriques à partir de données expérimentales. Masson; 1994. 